\title{
The Design and Implementation of Subway Locomotive Fault Diagnosis System Based on Adaptive Particle Filter
}

\author{
Zhenhua Wang ${ }^{1, a^{*}}$, Zhentao Liu ${ }^{1, b}$ and Linna $\mathrm{Xu}^{1, \mathrm{c}}$ \\ ${ }^{1}$ College of electrical and information, Dalian Jiaotong University, China \\ a1126705914@qq.com, b496186884@qq.com, c467191255@qq.com
}

Keywords: Subway locomotive; Adaptive particle; Labview; Fault diagnosis; Neural network

\begin{abstract}
Targeted at general testing process and index of multiple types of subway locomotive, combining the reality of subways in Dalian, this paper used adaptive particle filter and labview to design a multipaneled detection system for subway running. Actual survey shows that inner race fault and rolling bearing fault are two main reasons that cause faults of subway locomotive, based on these above, this paper use labview technology, mainly designing the signal waveform graphs based on TVAR model, GARCH model and graphs after filtering, so as to keep a real-time monitoring of subway running conditions. It matches with Neural network to analyze real-tome datas and give a result of fault diagnosis quickly.
\end{abstract}

\section{Introduction}

Subway malfunctions may cause by faults of electronic control system, hydraulic control system and some machinery parts inside the subway, these parts are complex singly and possible reasons and areas of troubles are complicated, so traditional technology is limited facing with these problems. By relative literatures we learn that subway locomotive system is a sophisticated, nonlinear and non-Gaussian system, so the traditional diagnostic method based on data and linear model is not suitable. Based on these above, my paper came up with a subway malfunction diagnostic method using adaptive particle filter and labview. Neural network is unique because it is fault tolerant, associative, presumable, mnemonic, self-adaptive and self-learning, it also can handle complex patterns, so it's important to combine labview and neural network technologies to research how to diagnose faults without disassembling. Therefore, a diagnosis system based on labview is designed to make both offline and online diagnosis for subway locomotives, and experiment shows that the system is accurate and fast to achieve the expected design goal [1].

\section{Hybrid Dynamical System's Faults Diagnosis Based on Particle Filter}

Generally, hybrid dynamical system is a complicated system, which is discrete but consecutive, highly nonlinear and non-Gaussian. Relative literatures show that the breakdown subway locomotive system is a typical hybrid dynamical system, including discrete failure modes and consecutive state variables [2].There are different structure and parameter between different failure mode and normal mode, it could be leap or continue. For the features of hybrid dynamical system, many experts explored the fault diagnosis based on adaptive particle filter. Generally speaking, particle filter is a random (Monte Carlo) way to monitor dynamical system, through weighting sample (particle) collection's probability distribution which is non-parameter, particle filter give us a method to estimate hybrid nonlinear system, which is computationally feasible and not controlled by Gaussian assumption (can show any distributions). Thus, it's the most common way to make fault diagnosis for complex systems recent years.

As normal operation of subway is vital to thousands of people's lives, the most basic requirement for subway diagnosis is accuracy, so I have to discuss the optimization way to improve particle filter's efficiency and accuracy. In present, ways we can use are as follows: optimizing sample method, adapting dimension-reducing and easing sample dilution technique, adjusting practical number 
automatically, improving according to the actual condition and so on. My article used optimizing sample method and designed adaptive particle filter to improve the efficiency and accuracy of the system.

\section{Hybrid Dynamical System's Faults Diagnosis Based on Particle Filter}

Identify Subway's Fault Signal Modes. BP (Back Propagation) neural network was put forward by an expert team leading by Rumelhart and McCelland in 1986.It's a feed forward neural network practiced by back propagation algorithm, is one of neural network modes applied widely. BP network can storage plenty of input - output Model mapping relations and don't need to reveal the mathematical equations that describe the mappings in advance. The learning rule of BP is using the steepest descent method, adjusting synaptic weights and bias of network through back propagation to minimize the sum of squared errors. Based on the above introduction, this paper adapted BP neural network technology, which consists of forward propagation and back propagation of information. Each unit of input layer is in charge of accepting outside information and deliver them to the middle layer, the middle one is Internal information processing layer, charging information conversion, according to the needs of information conversion, the middle layer can be designed to one or multiple hidden structures. The last hidden layer accepts the information from units of output layer, after further operation, one forward propagation is finished, and the output layer will deliver the result outside[3]. When real output is inconsistent with expectation, it comes to error back propagation, through output layer, weight of each layer is corrected by gradient descent way, and back propagated layer by layer, end-to-end back and forward propagation is the neural network training process, it can adjust weight of each layer and decrease the error to an accepted degree or expected learning times.

Subway Locomotive Diagnosis Formulas. As there are lots of modes of subway locomotive and varieties types of faults, the words of article are limited, it's impossible to list all aspects, so I just take examples of inner ring, outer ring and ball modes. When corrosive pitting happens the three above, a hit of certain frequency is produced, which cause vibration of subway locomotive, and machine running will have a periodic pulse, this pulse is very short and steep, different defective parts of subway would make a different characteristic frequency. There are some formulas of defective frequency:

When there are defects in inner race:

$$
\mathrm{f}=\mathrm{z} \frac{\mathrm{f}_{\mathrm{r}}}{2}\left(1+\frac{\mathrm{d}}{D}\right)
$$

When there are defects in outer race:

$$
\mathrm{f}=\mathrm{z} \frac{\mathrm{f}}{2}\left(1-\frac{\mathrm{d}}{D}\right)
$$

When there are defects in roller:

$$
\mathrm{f}=\mathrm{z} \frac{\mathrm{f}_{\mathrm{r}} D}{2 \mathrm{~d}}\left(1-\left(\frac{\mathrm{d}}{D}\right)^{2}\right)
$$

“d" is roller diameter, " $D$ " is medium diameter,"fr" is spindle rotation frequency, " $z$ " is the number of rollers, we can figure out the characteristic frequency of errors in inner race, outer race and rollers by measuring the size of subway locomotive[4].

It is a kind of 6207 deep groove ball metro locomotive, with the inside diameter of $35 \mathrm{~mm}$, the outside diameter of $72 \mathrm{~mm}$, the width of $17 \mathrm{~mm}$, the pitch diameters of $53.5 \mathrm{~mm}, \mathrm{~d}=18.5 \mathrm{~mm}, \mathrm{z}=9$, $\mathrm{n}=1450 \mathrm{r} / \mathrm{min}$. It was calculated that the fault frequency (characteristic frequency) of the inner raceway is $39.12 \mathrm{HZ}$, that of the outer raceway is $146.35 \mathrm{HZ}$, and that of the rolling element is $71.12 \mathrm{HZ}$.

Design of the Overall Function Chart. A complete fault diagnosis system of metro locomotive should include data acquisition and fault diagnosis. The former part is used to collect and storage vibration signals of the metro locomotive, while the latter processes the signals continuously or 
discontinuously to achieve fault diagnosis and get the result. In related literature, researchers collect vibration signals, detailedly classify them on the basis of vibration direction and generation mechanism, analyze characteristics of various signals and apply related signal processing and recognition technology to fault type recognition, which have achieved good results. The overall functional frame diagram of the system is shown in Fig. 1.

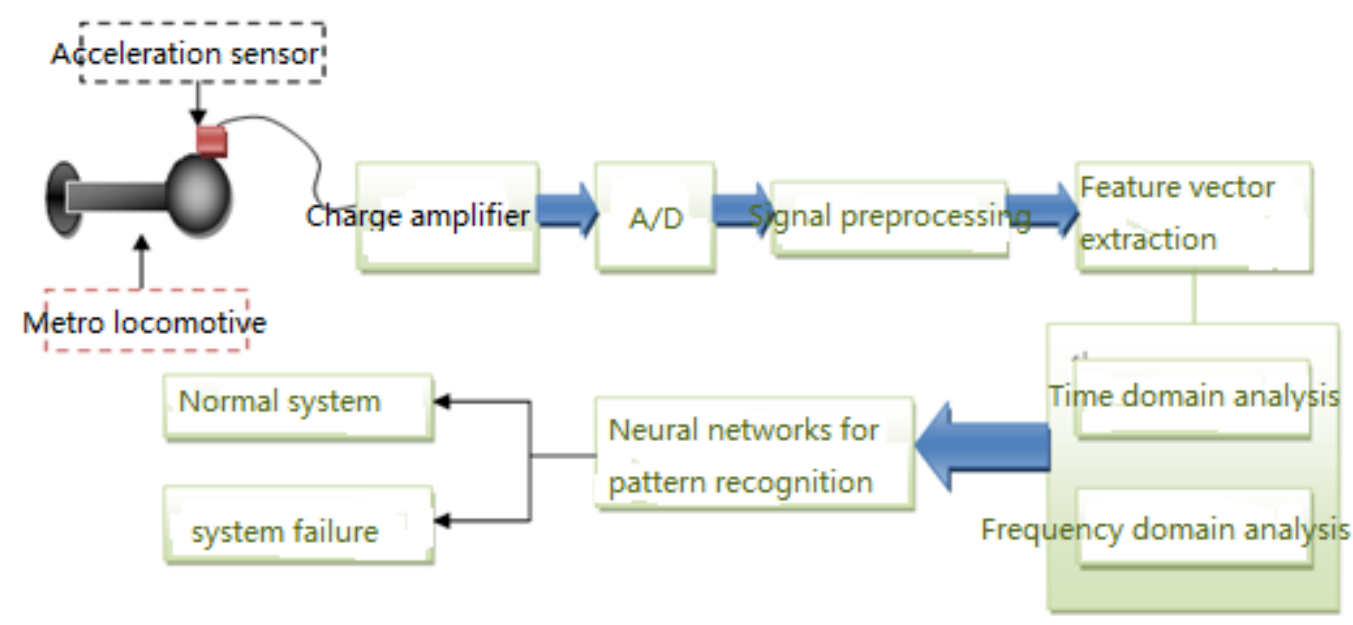

Figure 1. Overall functional frame diagram of the system

The data acquisition system (DAQ card) is composed of acceleration sensor, charger amplifier, $\mathrm{A} / \mathrm{D}$ converter and computer. In order to verify the correctness of the system design, researchers experiment with corresponding peripherals hardware circuits. The present experiment uses the acceleration sensor, which is the key part of the test technologies. Charger amplifier is consist of charger converter, adaptive adjustment, low-pass filter, high-pass filter, final power amplifier and power source. It is a kind of broadband one whose output voltage is proportional to the quantity of input electric charges [5]. And it can measure various mechanical quantities, such as vibration, concussion, pressure etc., connected with piezoelectric sensor. Features of it include converting mechanical quantity into proportional weak charge $\mathrm{Q}$ and extremely high output impedance Ra.

The acceleration sensor is placed on the experimental device of metro locomotive to acquire vibration signals that can be amplified by charge amplifier and then transformed into signals, which computer could identify, by A/D converter. Then, time domain graph of signals is drawn by MATLAB, which can be transformed into spectrogram by Fourier transform, from which the time domain and frequency domain eigenvalues can be extracted. In the end, pattern recognition is achieved by BP neural network to judge the fault type. The data acquisition card PCI-6251 need to be connected to acquire real time fault data during acquisition.

Setting up System. The overall labview program and system interface are shown in Fig. 2 and 3.

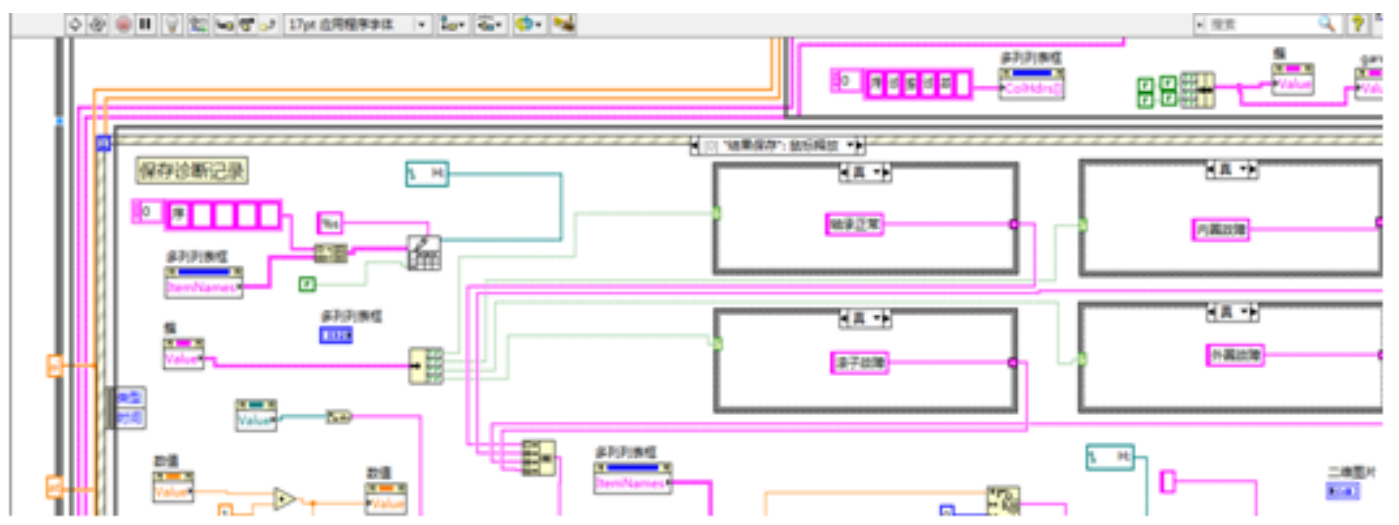

Figure 2. Parts of the labview program 


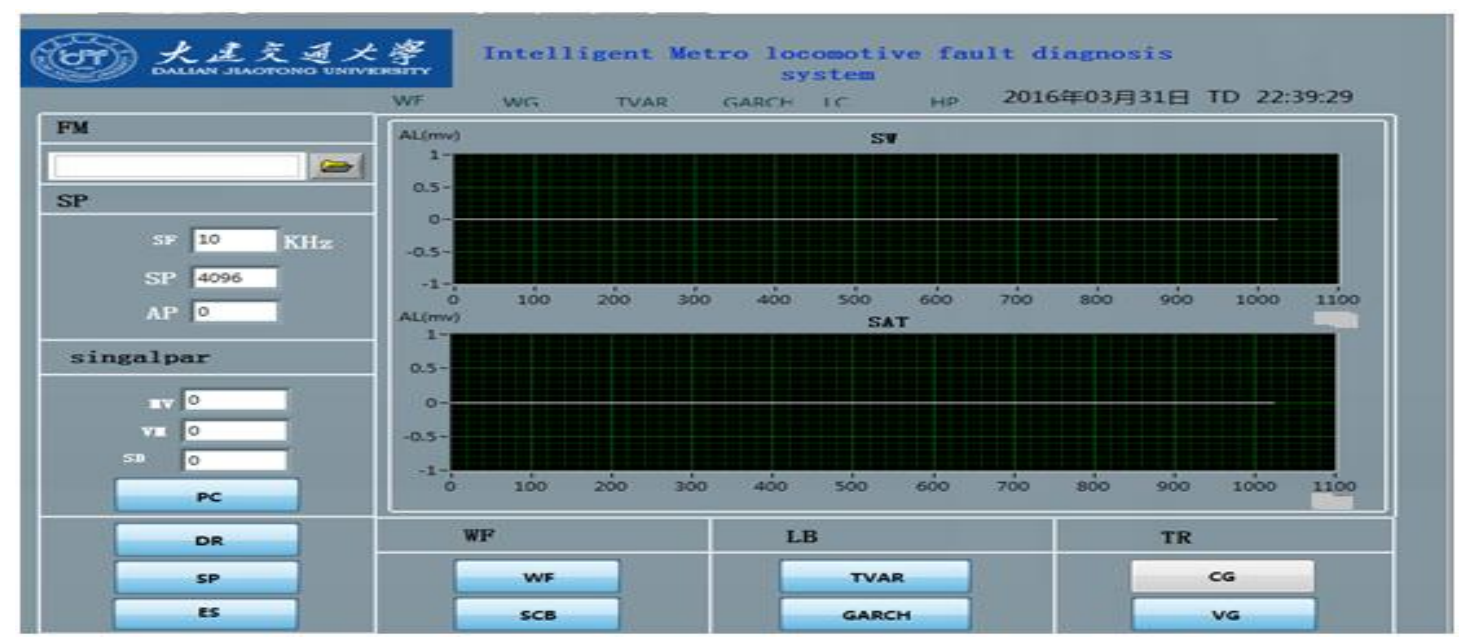

Figure 3. The operating interface of the system

In practical work, the system collects original fault signals (some system parameters, such as sampling frequency, sampling spot, analysis point are set up first) through data acquisition card (DAQ card) (the PCIe-6251 of NI Company, 24 analogy input resolution, the highest sampling frequency of $1.25 \mathrm{MS} / \mathrm{s}, 24$ channel analogy input), filters original signals, calculates relevant parameters (mean value, variance, standard deviation). Then system PC displays oscillograph after filter and draws diagnosis conclusion. It can choose TVAR model or GARCH one, meet various circumstances and accuracy requirement [6]. The system supports offline and online diagnosis, arrives real time diagnosis conclusions and auto-saves them. In order to improve its stability and accuracy, acceleration magnitude is needed to acquire. Thus CA-YD189 acceleration sensor is chosen, whose concrete parameters include sensitivity of $1000 \mathrm{mv} /(\mathrm{m} / \mathrm{s} 2)$, frequency range of $0.2 \sim 3 \mathrm{kHz}$, measuring range of $20 \mathrm{~m} / \mathrm{s}^{2}$, shock resistance of $500 \mathrm{~m} / \mathrm{s}^{2}$, because it is one of the most common sensors used in rotating machines. And it features durability, compact structure, low-weigh and wide frequency response. Accelerometer is widely used in many condition monitoring, which is installed on the metro locomotive seats via magnetic method. Metro locomotive produces high vibrational frequency during detection. In addition, the signal coordination instrument (SCXI), portable signal conditioning module(SCC) of the NI Company, is connected with data acquisition card(DAQ card) through SC-2345 shielding box.

\section{System Implementation}

Signal Filtering Implementation. In testing the original signal by the data acquisition need to preprocess before analysis, which would improve the reliability and authenticity of the data. And to check the randomness of the signal, in order to correctly select analysis method. In this design, we would adopt zero-mean process.

Zero-mean process also known as centralization treatment. Signal average is equivalent to a dc component, and the Fourier transform in direct current signal is the impulse function on $\omega=0$. So if we do not remove the mean, it will have a large spectrum peak present to $\omega=0$, in the signal spectrum analysis. And it also effect spectrum curve on the value of $\omega=0$ this makes it have a large error. In conclusion, we need to carry on filter processing to the original signal. The picture above is the original signal [7]. 


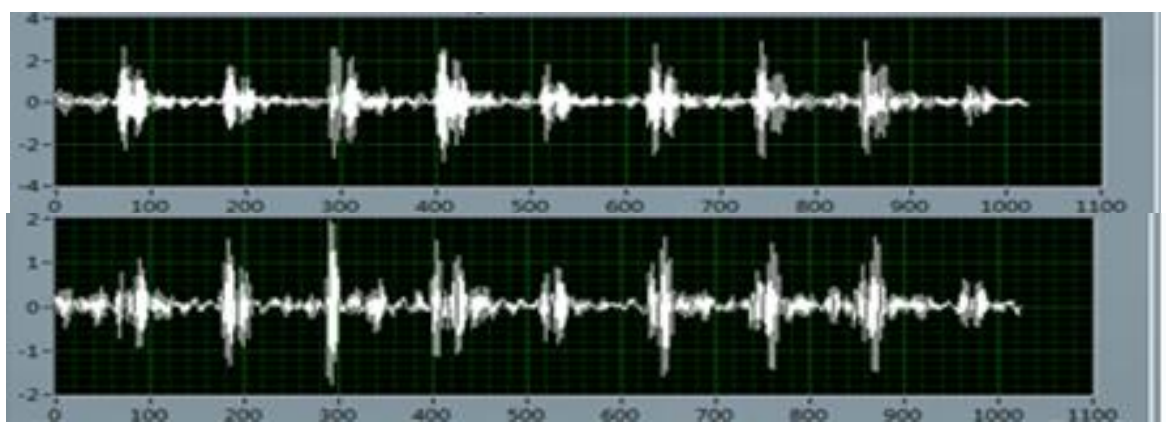

Figure 4. Original signal filter processing contrast

The Implementation of Neural Networks in TVAR model. When implementing this module, we should use matlab script which is labview and matlab joint debugging. The main function is about neural network training system and characteristic value extraction which under the condition of giving samples in the fault diagnosis [8]. The samples of fault diagnosis mainly aims at the inner-race faults, out-race faults and roller faults. In neural network training, the system fault diagnosis accuracy rate can reach the set value within a short period. Tthe waveform of the training process by matlab script could be generated in matlab environment. The system interface implementation and training process waveform were shown in Fig. 5.

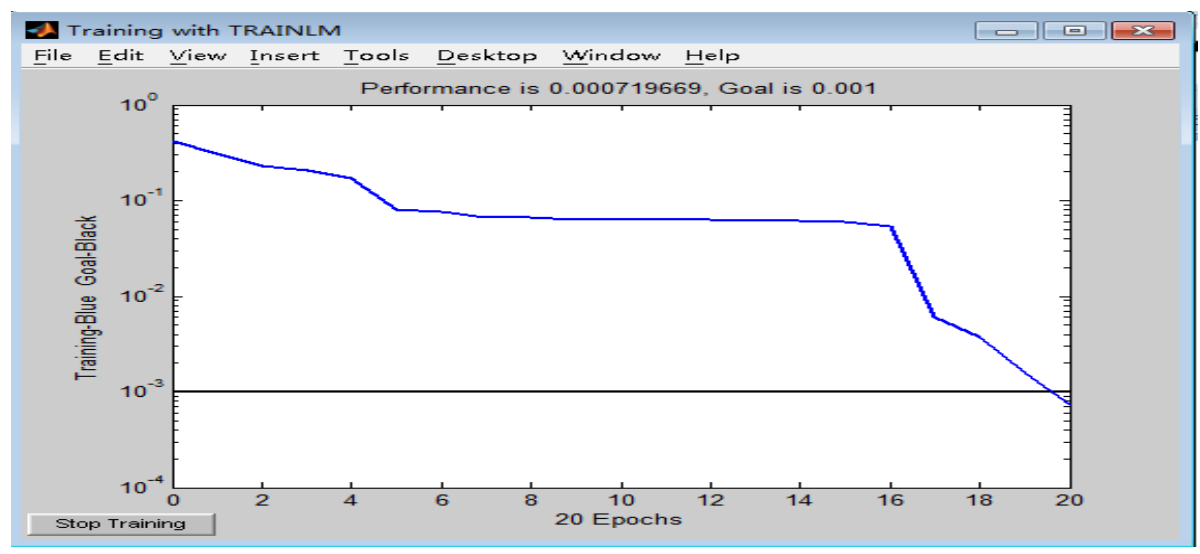

Figure 5. Training process accuracy changes of waveform

Labview has a strong and fast control chart editing function, matlab has powerful mathematical modeling emulation facility[9]. In order to combine the advantages of them, this system adopts the embedded matlab script in labview environment to realize the alignment of them.

The Fault Samples of the Output Waveform. In order to effectively troubleshoot the fault diagnosis performance of system, the system has been totally set up 12 samples, 6 of them are normal, and rest 6 samples are faulty, the specific list as shown in Table 1.

Table 1 can be learned that the system would quantify the value on three characteristics in the frequency domain (center frequency, frequency deviation, mean square frequency), and then designate a characterization system normal range by determining whether the value of the quantization interval is normal which could determine the fault existence, the fault type is determined by the characteristics of the type of quantized values abnormalities [10]. Read module system with the system's own document, combine the previous trained neural network samples, the relevant system output waveform shown in Fig. 6. 
Table 1 Samples for testing

\begin{tabular}{|c|c|c|c|c|}
\hline Condition & Sample & \multicolumn{3}{|c|}{ Frequency Domain } \\
\cline { 3 - 5 } & & $\begin{array}{c}\text { Gravity } \\
\text { Frequency }\end{array}$ & $\begin{array}{c}\text { Frequency } \\
\text { Variance }\end{array}$ & MSF \\
\hline \multirow{4}{*}{ Malfunction } & G2015 & 297.5159 & 858500 & 947020 \\
\cline { 2 - 5 } & G2515 & 648.5740 & 1643900 & 2064500 \\
\cline { 2 - 5 } & G3015 & 708.4801 & 1753300 & 2255200 \\
\cline { 2 - 5 } & G20610 & 324.1613 & 926720 & 1031800 \\
\cline { 2 - 5 } & G25610 & 635.1468 & 1618300 & 2021700 \\
\cline { 2 - 5 } Normal & G30610 & 601.0822 & 1552000 & 1913300 \\
\cline { 2 - 5 } & Z2015 & 485.0321 & 1307500 & 1542800 \\
\cline { 2 - 5 } & Z2515 & 527.8103 & 1401900 & 1680500 \\
\cline { 2 - 5 } & Z3015 & 518.8398 & 1382000 & 1651200 \\
\cline { 2 - 5 } & Z20610 & 540.0254 & 1427300 & 1718900 \\
\cline { 2 - 5 } & Z25610 & 516.9125 & 1378200 & 1645400 \\
\hline \multirow{7}{*}{} & Z30610 & 516.5208 & 1377100 & 1643900 \\
\hline
\end{tabular}

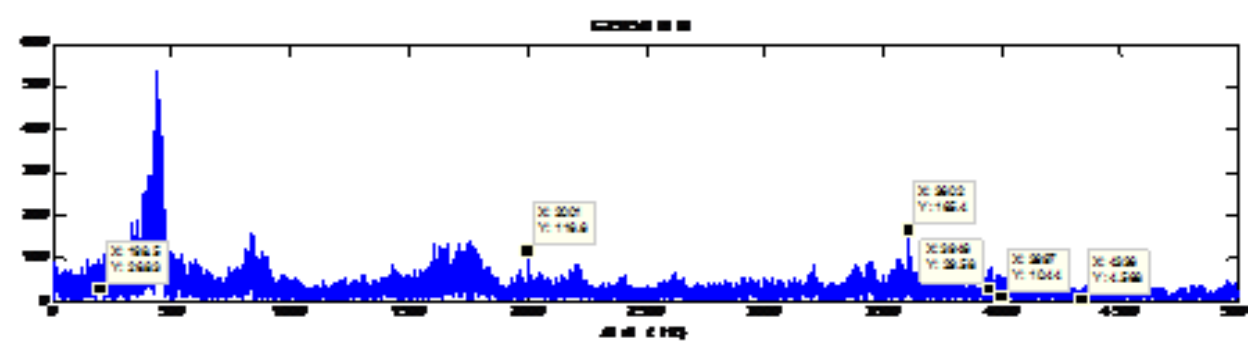

Figure 6. The fault samples of the output waveform

\section{Summarize and Expectation}

The system using adaptive particle filter which is a powerful tool, to design high precision of metro locomotive fault diagnosis system. With a large number of samples of neural network training, the basic system has the function of similar pattern recognition and fault diagnosis. By different types of fault sample tests show the system can better identify the type of fault. According to the waveform output in matlab script environment, the system can achieve the setting identification accuracy in a short period during neural network training. By analysis the waveform in the normal samples and the fault samples, system would own high accuracy and fast speed, which also can achieve the expected goal. In addition, the system in the labview development environment, user-friendly, simple operation and suitable for practical popularization and application.

\section{References}

[1] Zhang Taiyuan. The Implementation of Underground Locomotive Condition Monitoring and Fault Diagnosis System Based on LabVIEW [J]. Academic Journal of Beijing Jiaotong University, 2014, 6.

[2] Ge Xinfeng, Wang Zhi Hao, Gu Qing, Tang Shu. Subway Locomotive Fault Diagnosis Based on Signal Analysis [J]. Data Acquisition and Processing, 2010, 12.

[3] Liu Xuejiang, Liu Haisheng, Wang Huigang. Fault Diagnosis System for Metro locomotive based on LabVIEW [J]. Coal Mine Machinery, 2011, 1. 
[4] Zhu Keheng. The Extraction and Diagnosis Methods of Subway Locomotive on Vibration Signal Feature [D]. Dalian University of Technology, 2013,10.

[5] Xiao Wenbin. The Evaluation Research on Metro Locomotive Fault Diagnosis and Performance Dassessment of Based on Coupled HMM [D]. Shanghai: Shanghai Jiao Tong University, 2011

[6] Li Li, Wang Hongmei. Diagnosis Research of Rolling Fault Based on Coupled HMM [J]. Metro locomotive, 2012, 6: 42-45.

[7] Fu Fu, Zhang Youyun, Zhu Yongsheng. Case-based Reasoning Method for Fault Diagnosis of Metro Locomotive [J]. Academic Journal of Xi'an Jiao Tong University 2011, 45 (11): 79-84.

[8] Li Yunlai, Wang Chieh, Zhu Hao. The Methods Overview of Fault Detection Based on Virtual Instrument Technology [J]. Academic Journal of Southwest Jiao Tong University, 2014, 23 (18): 23-25.

[9] Li Wei, Zhu Qiannan. Analysis Method of Locomotive Metro Fault [J]. Academic Journal of Tongji University, 2013, 34 (12): 26-31.

[10] Li Hongcai, Zhang Dawei. Detection Method for A New Type of Subway Locomotive based on virtual instrument [J]. Modern electronic technology, 2013,11 (25):12-15. 